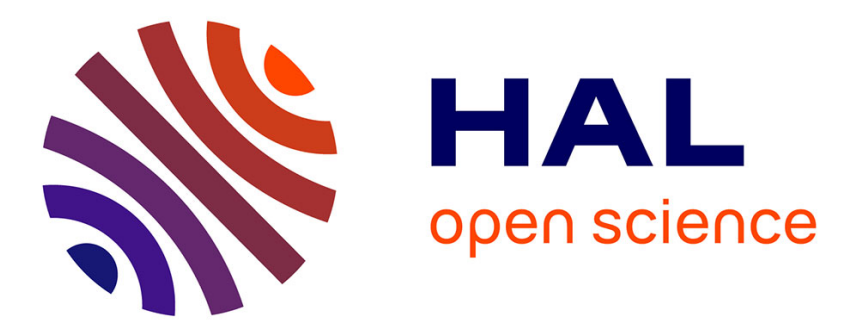

\title{
Perception of gaze direction in glaucoma: a study on social cognition
}

Aude Denoyelle, Jean-Francois Rouland, Naima Goual, Muriel Boucart

\section{To cite this version:}

Aude Denoyelle, Jean-Francois Rouland, Naima Goual, Muriel Boucart. Perception of gaze direction in glaucoma: a study on social cognition. Optometry and Vision Science, 2020, 10.1097/OPX.0000000000001496 . hal-03018196

\section{HAL Id: hal-03018196 https://hal.science/hal-03018196}

Submitted on 30 Nov 2020

HAL is a multi-disciplinary open access archive for the deposit and dissemination of scientific research documents, whether they are published or not. The documents may come from teaching and research institutions in France or abroad, or from public or private research centers.
L'archive ouverte pluridisciplinaire HAL, est destinée au dépôt et à la diffusion de documents scientifiques de niveau recherche, publiés ou non, émanant des établissements d'enseignement et de recherche français ou étrangers, des laboratoires publics ou privés. 


\section{Perception of gaze direction in glaucoma: a study on social cognition}

Aude Denoyelle ${ }^{1}(\mathrm{MD})$, Jean François Rouland (MD-PhD) ${ }^{1-2}$, Naima Goual $(\mathrm{MSc})^{2}, \&$ Muriel Boucart $(\mathrm{PhD})^{2}$

1. Centre Hospitalier Universitaire de Lille, Hôpital Huriez, service d'ophtalmologie, Lille, France

2. SCALab, University of Lille, CNRS, France.

Corresponding author: Muriel Boucart, Faculté de médecine de Lille, Pole recherche, 1 place de Verdun, 59000 Lille, France. E-mail : muriel.boucart@ chru-lille.fr

Running head: perception of gaze direction in glaucoma

Key words: glaucoma, face perception, gaze direction, central vision

Financial support: none

ABSTRACT

Significance: Discriminating quickly where another person's gaze is directed is a key component of social interaction, as gaze direction conveys information about others' intentions (approach or avoidance) and shift in gaze is used in group conversation. This study shows that patients with glaucoma are delayed in their discrimination of gaze direction.

Purpose: To investigate whether glaucoma affects the perception of gaze direction.

Methods: 24 patients with open-angle glaucoma, 24 age-matched controls and 20 young normally sighted controls were presented with faces displayed centrally for $200 \mathrm{~ms}$. The gaze could either be direct or averted and the head could be a frontal view or a rotated view. Half of the participants in each group were asked to press a key only for faces with a direct gaze. 
31 The other half responded for a face with an averted gaze. The orientation of the head had to be

32

33 ignored.

Results: Accuracy was above $90 \%$ correct for all three groups. We found no difference in performance between young and older controls, except for shorter response times for the frontal view than for the rotated face in young participants. Patients with glaucoma needed on average $140 \mathrm{~ms}$ longer to decide if the gaze was averted than for the direct gaze and they were less accurate than controls in perceiving the gaze as direct when the head was rotated.

Conclusions: Patients with glaucoma often experience impaired vision due to a reduced sensitivity in central vision. Although lower central sensitivity had little effect on their ability to discriminate gaze orientation, they required on average $140 \mathrm{~ms}$ longer than age-matched controls to perceive an averted gaze.

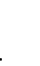

45

$$
6
$$

(1)



glaucoma also exhibit reduced visual acuity and reduced contrast sensitivity in central vision. ${ }^{1-2}$ Their vision becomes more degraded with the progression of the disease, impairing daily life activities such as reading and driving. ${ }^{3-4}$ Patients with glaucoma also report difficulties in recognizing people in the street. ${ }^{5}$ In a series of experiments designed to measure the threshold distance for recognition of gender, facial expression and identification of famous people in central vision, we have shown that participants with glaucoma required faces to be substantially closer (i.e., larger) than normally sighted age-matched controls, in order to compensate for their reduced contrast sensitivity. ${ }^{6}$

Faces have an important social function as they convey information about gender, age, emotional state and identity. Within the face, the eye region is the most attended part and the most frequently fixated region. ${ }^{7}$ The attraction towards the eye region starts early. Newborns look longer at faces whose gaze is directed at them than at faces with averted gaze. ${ }^{7}$ In adults, gaze perception plays a critical role in social and non-verbal communication. In everyday life, gaze direction conveys information about others' intentions, signaling one's intention to approach and engage communication (direct gaze) or to avoid a person (averted gaze). ${ }^{8}$ Gaze direction also signals whether the person is attending to you (direct gaze) or to something or someone else (averted gaze). During conversation, changes between direct and averted gaze are used to regulate turn-taking. ${ }^{9}$ Gaze is interpreted automatically and effortlessly when perceptual processes are reliable.

As the eye region is a key element for inter-individual interactions, a reduced ability to perceive gaze direction may have a negative impact on social cognition, for instance in altering the interpretation of others' intentions. Misinterpretation of social signals undermines social interactions and can be associated with stress, anxiety or social withdrawal. For instance, Torres-Marín et al. ${ }^{10}$ showed that individuals with a high degree of gelotophobia (a 
personality trait characterized by a disproportionate fear of being laughed at by others) make more errors when identifying gaze direction. They avoid others as they expect contempt and rejection.

Studies on normally sighted young observers have shown that gaze direction is a critical social cue in human communication. Indeed, faces looking directly at the perceiver are rated as more attractive and more trustworthy ${ }^{11}$, while gaze avoidance is perceived as a sign of insincerity in Western culture. ${ }^{12}$ Smiling faces with a direct gaze are rated as happier than smiling faces with an averted gaze, and the perceived intensity of anger is greater with an angry face gazing directly than with an averted gaze. ${ }^{13-14}$ A person's direction of gaze (direct or averted) has an impact on his or her subsequent memorability. ${ }^{15}$ In visual-search paradigms, starring faces pop out when embedded within distractor faces with averted gaze. ${ }^{16-}$ ${ }^{17}$ Moreover, search efficiency is almost independent of the number of distractors when the target is a face with direct gaze. ${ }^{16-17}$

In the present study, we explored the ability of patients with glaucoma to perceive the social signal of gaze direction. Participants with glaucoma and normally sighted young and older controls were presented with direct or averted gaze in faces whose heads were either displayed frontally or turned $30^{\circ}$ to the left. They were asked to categorize gaze direction regardless of head orientation. We hypothesized that reduced central sensitivity, even though not yet visible by perimetry, would affect perception of gaze direction in terms of accuracy and response times in participants with glaucoma, particularly for rotated heads. Indeed, it has been shown that performance on categorization of gaze direction is better for facing heads than for rotated heads in regions of reduced acuity and reduced contrast sensitivity (in peripheral vision) in normally sighted participants. ${ }^{18}$ We also expected patients with a central visual field defect to be more impaired than patients with no central visual field defect. 
$\underline{\text { Participants }}$

The characteristics of the participants are presented in Table 1 for patients and in Table 2 for controls. Twenty-seven patients who consulted in the department of ophthalmology in Lille University Hospital agreed to participate in the experiment. Three were excluded from the analyses due to glaucoma at an end stage. Twenty-four patients (11 males) with visual field (VF) defects in both eyes due to primary open-angle glaucoma were kept for the analysis of data. They ranged in age from 35 to 85 (mean 64.9). Each patient received full ophthalmological work-up including a visual field evaluation just before the experiment. Visual field sensitivity (expressed as mean deviation: MD) was measured with a Humphrey Field Analyzer (HFA, Carl Zeiss Meditec, CA, USA). The 30-2 technique (SITA standard) was used for all patients. All patients exhibited a bilateral visual field defect. Nine of the patients exhibited a unilateral or a bilateral central visual field defect at the 30-2 visual field. These patients were tested with a 10-2 visual field (examining central vision more finely) in addition to the 30-2 visual field test. The visual fields (30-2 and 10-2) were measured monocularly. The visual field was considered as reliable if the rate of false positives or false negatives was lower than $20 \%$ and the number of fixation losses was less than $33 \%$. To estimate the binocular visual field of patients, an integrated visual field (IVF) was built from the two monocular (left and right eye) visual fields. ${ }^{19-20}$ Sensitivity values for each point in the IVF were computed by taking the maximum sensitivity from corresponding points in the left and right eye visual fields. The IVF was generated with a bespoke computer program written in MATLAB (MATLAB R2017a (MathWorks, Inc., Natick, MA, USA). The IVFs are presented in Figure 1. 
Twenty-four age-matched controls (13 males) ranging in age from 35 to 80 (mean: 62.2) were recruited among the patients' relatives. The young controls were 20 medicine students (14 males) ranging in age from 21 to 30 (mean 27.1). Both young and older participants had received an ophthalmological examination during the 6 months preceding inclusion without any sign of glaucoma. An evaluation of their binocular visual acuity (Snellen chart) was performed again just before testing. All participants were tested with their habitual optical correction. For all participants, the criteria of inclusion were a binocular visual acuity equal to or better than $8 / 10$ (Snellen equivalent), no history of neurological or psychiatric disease, no chronic use of drugs that can alter attention (benzodiazepines), no ocular disease, no family history of glaucoma for controls and no other ocular disease than glaucoma (e.g., macular degeneration or cataract) for patients. In addition, all the patients and the age-matched controls older than 60 were assessed with the French version of the Mini Mental State Examination (MMSE) to check for cognitive impairment. Participants whose MMSE score was lower than 25/30 were excluded from the study. Patients and age-matched controls did not differ significantly in age $(\mathrm{t}(46)=0.82, \mathrm{p}=0.41)$. We also compared older patients and controls on the MMSE score. As the condition of normality was not fulfilled, a non-parametric test (Mann-Whitney) was used. The two groups did not differ significantly on their MMSE score $(\mathrm{U}=125, \mathrm{p}=0.735)$. The study was approved by the ethics committee of the University of Lille (RVPG 2018 - 270-60). In accordance with the tenets of the Declaration of Helsinki, written informed consent was obtained from all participants. 
Stimuli: The stimuli were 20 colored photographs of young male and female faces, displaying neutral expression, provided by $\mathrm{N}$ George. ${ }^{21}$ Each face was photographed in four versions determined by the orientation of head (frontal view vs. head rotated $30^{\circ}$ towards the left), with the eyes looking straight ahead at the camera (direct) or $30^{\circ}$ to the right (averted). Examples of the four versions are shown in Figure 2. Each face was displayed centrally on a black background screen. We measured the luminance of the faces using a photometer (Minolta CS 100). The measurements were made in the same lighting conditions as those of testing. The photometer was focused on the nose. The mean luminance of the female faces was 29.02 $\mathrm{cd} / \mathrm{m} 2$ (SD: $10.1 \mathrm{~cd} / \mathrm{m} 2)$. The mean luminance of the male faces was $26.1 \mathrm{~cd} / \mathrm{m} 2$ (SD: 8.2 $\mathrm{cd} / \mathrm{m} 2)$. The luminance of the background was $2.4 \mathrm{~cd} / \mathrm{m} 2$.

Procedure: Participants were seated at a viewing distance of $120 \mathrm{~cm}$ from a screen (Dell 30 inches; Dell Computer Corporation, Texas, USA). The resolution of the screen was $2560 \mathrm{x}$ 1600 pixels. At a viewing distance of $120 \mathrm{~cm}$, the angular size of the screen was $30.56^{\circ}$ horizontally $\mathrm{X} 19.1^{\circ}$ vertically. The stimuli were presented under photopic conditions, the light coming from above without reflecting on the screen. The software was written in Matlab (MathWorks, Inc; Natick, MA, USA) with the Psycho Toolbox. Following a central white fixation cross displayed on a black background for $500 \mathrm{~ms}$, a face $\left(6.25^{\circ}\right.$ x $\left.5^{\circ}\right)$ was presented centrally on the screen for $200 \mathrm{~ms}$. It was followed by a random inter-stimulus interval between 1000 and $2000 \mathrm{~ms}$, during which the black background was displayed. A go / no go paradigm was used. A target was defined at the beginning of the experiment: direct gaze for half of the participants in each group and averted gaze for the other half. Participants were asked to press a key when they saw the target, regardless of head orientation, and to refrain from responding when the gaze direction did not correspond to their pre-defined target. Prior to the experiment, participants were presented with an example of the four versions of a face (frontal direct, frontal averted, rotated direct and rotated averted) on paper, and their target 
(direct or averted gaze) was shown. This was followed by 20 practice trials on faces that were not part of the experimental set. The practice session was followed by 80 trials ( 20 faces $\mathrm{X} 2$ gaze directions X 2 head orientations).

\section{Statistical analysis}

Statistical analyses were conducted with the software Systat 8 (Systat Software, Inc; San Jose CA, USA). Two ANOVAs were conducted, one on accuracy (hits) and one on response times. Group and gaze direction were the between factors. Head orientation was the within factor. The results are presented in Figure 2. The Spearman $\mathrm{R}$ test was used to check for any relationship between performance and age and, for patients, between performance and the mean deviation of the IVF.

$\underline{\text { Accuracy }}(\mathrm{CI}=$ Confidence Interval 95\%)

On average, accuracy was high and did not differ significantly between the three groups (young: 97.25\% (CI upper $=100 \%$ CI lower: 91.3\%), age-matched: $94.75 \%(\mathrm{CI}$ upper $=$ 99.1\% CI lower: $89 \%$ ), patients: $93.5 \%(\mathrm{CI}$ upper $=98.4 \% \mathrm{CI}$ lower: $88.6 \%) ; \mathrm{F}(2,65)=0.99$, $p=0.37)$. A deficit in the upper part of the IVF (P5, P6, P10, P13, P14, P21) did not affect performance (97.1\%). Patients with a unilateral central field defect (P9, P15, P16, P24) did not differ from the age-matched group (94\%) but the four patients with a bilateral central field defect (P1, P6, P13, P23) exhibited a lower accuracy (90.5\%). There was no main effect of gaze direction $(\mathrm{F}(1,65)=0.03, p=0.8)$. Head orientation affected performance with a higher accuracy for the frontal view than for the rotated head $(97.6 \%$ vs. $92.75 \% ; \mathrm{F}(1,65)=3.8, p$ <.05). A three-way interaction was observed between group, head orientation and gaze 
direction $(\mathrm{F}(2,65)=3.27, p<.044)$. A separate analysis for each group showed that this interaction resulted mainly from the patients' group. Indeed, no main effect of head orientation $(\mathrm{F}(1,40)=1.10, p=0.30)$ and gaze direction $(\mathrm{F}(1,40)=0.62, p=0.43)$ was observed in the young and in the age-matched groups. In the patients' group, the analysis showed a main effect of gaze direction, with a significantly better performance for averted than for direct gaze $(96.5 \%$ vs. $90.5 \% \mathrm{~F}(1,22)=5.4, p<.03)$, a significantly better accuracy for the frontal face than for the rotated face $(96.25 \%$ vs. $90.75, \mathrm{~F}(1,22)=4.3, p<.05)$ and a significant interaction between face orientation and gaze direction $(\mathrm{F}(1,22)=4.31, p<.05)$. As can be seen in Figure 2, this interaction resulted from the rotated face. Patients who were assigned the "direct gaze" as target pressed the key less often for their target when the face was rotated (rotated: $85 \%$ vs. frontal view: $96 \%, \mathrm{t}(13)=2.22, p<.04$ ).

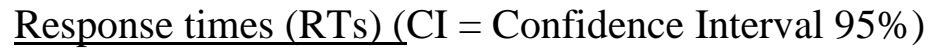

The results are presented in Figure 2. Though patients were, on average, slower than young and age-matched controls, the RTs did not differ significantly between groups (young: $548 \mathrm{~ms}$ (CI upper $=598$ ms CI lower: $497 \mathrm{~ms}$ ), age-matched: $547 \mathrm{~ms}(\mathrm{CI}$ upper $=616 \mathrm{~ms}$ CI lower: $478 \mathrm{~ms})$, patients: $591 \mathrm{~ms}(\mathrm{CI}$ upper $=671 \mathrm{~ms}$ CI lower: $511 \mathrm{~ms}) \mathrm{F}(2,65)=2.3, p=0.11)$. Patients with a unilateral central field defect were on average slower than patients with a bilateral central field defect (633 vs. $522 \mathrm{~ms}$ ). There was a significant main effect of gaze direction, with shorter RTs for direct than for averted gaze (527 vs. $597 \mathrm{~ms} \mathrm{~F}(1,65)=8.08, p$ <.006). RTs were shorter for the frontal view than for the rotated face but the difference failed to reach statistical significance (553 vs. $573 \mathrm{~ms}, \mathrm{~F}(1,65)=3.03, p<.08$ ). There was no significant interaction between group, gaze direction and head orientation. However, as we hypothesized that a lower sensitivity in central vision (not visible with a 30-2 visual field test) might affect perception of gaze direction in glaucoma, we conducted a separate analysis on 
the three groups to check if the patients behaved differently from the normally sighted participants. For young participants, the only main effect occurred for face orientation, with shorter RTs for the frontal view than for the rotated view of the face (529 vs. $566 \mathrm{~ms} \mathrm{~F}(1,18)$ $=16.6, p<.001)$. No significant effect of head orientation and gaze direction was found in the age-matched group. In the patients' group, the only main effect was that of gaze direction, with longer RTs for averted than for direct gaze (662 vs. $521 \mathrm{~ms} ; \mathrm{F}(1,22)=7.9, p<.01)$.

\section{$\underline{\text { Correlations }}$}

There was a significant association between age and head orientation in the patients' group, with a lower accuracy for rotated than for frontal head view in the older patients $(r=0.472$, $\mathrm{p}<.05$ ). No association was observed in the age-matched group (frontal view: $\mathrm{r}=0.207, \mathrm{p}=$ 0.332; rotated view: $\mathrm{r}=0.046 ; \mathrm{p}=0.833$ ). No significant association was observed between the MD of the IVF and accuracy (frontal view: $r=0.108 ; p=0.616$ vs. rotated head: $r=$ $0.036 ; p=0.867$ ) and between the MD of the IVF and response times (frontal view: $r=0.139$; $\mathrm{p}=0.517$ vs. rotated head: $\mathrm{r}=0.039 ; \mathrm{p}=0.857)$.

[Figure 2 about here]

\section{DISCUSSION} attention was explicitly directed to gaze direction. The main results were as follows: i) accuracy was high for all three groups; ii) participants with glaucoma needed more time to decide if gaze was averted than for direct gaze and they were more impaired in perceiving gaze as direct when the head was rotated; iii) no difference in performance was observed between young and older controls, except for shorter response times for the frontal view than for the rotated face in young participants. 

higher in the congruent condition of frontal head view and direct gaze. ${ }^{22}$ Though irrelevant for the task, the rotated head interfered with performance, increasing response times and decreasing accuracy. This was particularly marked for participants with glaucoma, suggesting that head orientation was processed more rapidly and interfered with the processing of gaze direction in patients. A possible explanation for this result is a decrease in sensitivity at higher spatial frequencies in central vision in patients with glaucoma. Vida and Maurer compared the spatial frequency tuning for judgments of eye gaze to the spatial frequency tuning for judgments of facial identity in normally sighted participants. ${ }^{23}$ They found that the visual system relies on higher spatial frequencies for judgments of eye gaze (approximately 20 cycles/face) than for judgments of facial identity (approximately 15 cycles/face). A priority for processing head orientation over gaze direction in peripheral vision was also reported by Florey and colleagues in normally sighted young participants. ${ }^{18}$ They found that head orientation determined whether the gaze was perceived as direct or deviated in peripheral vision (at $6^{\circ}$ and $9^{\circ}$ eccentricity) where sensitivity is reduced, whilst head orientation had no influence on categorization of gaze direction in central vision. As for spatial frequencies, ${ }^{23}$ the lower resolution of peripheral vision provides an advantage for coarse rather than to fine information (head before eye-gaze). Though irrelevant for the task, the effect of head orientation in the patients' performance supports the hypothesis of a lower spatial sensitivity in central vision in participants with glaucoma. Patients also exhibited longer response times for averted gaze. However, this was associated with better accuracy. This result is also in line with difficulties in discriminating gaze direction, likely due to reduced sensitivity in the perception of small details (the location of the iris in the sclera) in patients. 
Neuroimaging research has made progress in mapping out the networks and areas involved in face and gaze processing. Gaze processing involves a large network of brain areas, including anterior and posterior parts of the superior temporal sulcus (aSTS and pSTS), the lateral parietal cortex and the prefrontal cortex. The anterior part of the STS distinguishes different averted gaze directions in a head view-invariant manner. The posterior part is responsible for processing dynamic aspects of faces, such as gaze shift and facial expression. The lateral parietal cortex is involved in gaze-cued attention orienting and the prefrontal cortex is engaged when gaze following is used to establish joint attention. ${ }^{24-27}$ Studies on animal models of glaucoma and neuroimaging studies in humans suggest that the progressive destruction of retinal ganglion cells in glaucoma results in axonal loss in the optic nerve, chiasm and tract that can trigger trans-synaptic degeneration of the optic radiations and visual cortex. ${ }^{28}$ Evidence for anatomical and functional alterations within and beyond the visual system has been reported in patients with glaucoma during the last ten years. ${ }^{29}$ Structural (reduced cortical thickness and volume) and metabolic abnormalities have been found in the visual cortex and in higher level visual areas such as the fusiform gyrus, the cuneus and the middle temporal gyrus, with a positive correlation between thinning of the visual cortex and glaucoma severity. ${ }^{30-32}$ The occipito-temporal cortex contains regions that are tuned to face and gaze perception (the superior temporal sulcus, the occipital face area, the fusiform face area). ${ }^{26,33}$ In addition to perceptual impairment (e.g., the difficulty to perceive the iris), it could be that a dysfunction of STS affects gaze perception in participants with glaucoma.

\section{Except for shorter response times for the frontal view than for the rotated face in} young participants, no difference in performance was observed between young and older controls. Studies on normal aging on the perception of social cues suggest that older adults are less sensitive to gaze direction cues than young adults, at least when following gaze and 
299 judging the intensity of emotional expression, ${ }^{34}$ though this result is modulated by the age of 300 the face stimuli. ${ }^{35}$ This has been attributed to age-related neural decline in social skills rather than to age-related perception or attention deficits.$^{36}$

Conclusion and limitations: The current study highlights the impact of glaucoma on the perceptual aspects of social cognition. Detecting an averted gaze rapidly can be important in certain situations because a speaker's averted gaze can signal either deceptive intentions or that something or someone has captured the person's attention, which can sometimes represent a potential danger. Perceiving gaze direction adequately is important in social situations like group conversations. Difficulties in recognizing gaze direction, or a delayed detection of gaze shift, might reduce commitment in group discussions.

This study has several limitations. First, the 10-2 visual field, which measures sensitivity in the central visual field, was not collected in all participants with glaucoma. It was performed only in those at a severe stage who exhibited a central visual field defect on the 30-2 visual field test. Evidence is accruing to suggest that the macular region is impaired early in patients with glaucoma. The central visual field losses can be missed when testing with widely spaced test locations as used in the 30-2 visual field. ${ }^{37}$ Second, the faces were displayed for only 200 ms, whilst people can explore faces for an unlimited time in real life. Third, we did not measure the visual field sensitivity of age-matched controls and so we could not include their sensitivity as a co-variate in the analysis to determine if alterations in gaze perception were predictable from changes in gaze sensitivity. 
322

Table 1. Clinical and demographic data about patients. $\mathrm{RE}=$ Right Eye. LE $=$ Left Eye. MMSE $=$ Mini Mental State Evaluation. $\mathrm{IVF}=$ integrated visual field. $\mathrm{MD}=$ Mean Deviation: a Mean Deviation comprised between $-6 \mathrm{~dB}$ and $-12 \mathrm{~dB}$ is classified as moderate glaucoma. It is classified as severe glaucoma between $-12 \mathrm{~dB}$ and $-20 \mathrm{~dB}$. Under $-6 \mathrm{~dB}$, the glaucoma is at an early stage. The central visual field deficit was defined by a reduced sensitivity $(<5 \mathrm{~dB})$ in the central $10^{\circ}$ of the visual field. The 10-2 visual field was used only for patients at a severe stage of glaucoma, either on one eye or on both eyes. $\mathrm{N}=$ not measured.

Table 2. Demographic data about age-matched and young controls. Binoc VA = binocular visual acuity. MMSE = Mini Mental State Evaluation.

Fig.1. The integrated visual field of each patient. Patient 4's left visual field could not be measured.

Fig.2. Middle: an example of the four versions of each face determined by two head orientations (frontal view vs. rotated) and two gaze directions (direct vs. averted). A: mean accuracy (with standard errors) in the three groups (patients, age-matched controls and young controls) as a function of the two head orientations and the two gaze directions. B: mean response times in ms (with standard errors) in the three groups (patients, age-matched controls and young controls) as a function of the two head orientations and the two gaze directions. 
342 Acknowledgements: The authors are grateful to Pete R Jones for computing the integrated 343 visual fields from the monocular visual fields of all patients. 
1. Lenoble Q, Lek JJ, McKendrick AM. Visual object categorisation in people with 347 glaucoma. Br J Ophthalmol. 2016;100:1585-90.

2. Roux-Sibilon A, Rutgé F, Aptel F, et al. Scene and human face recognition in the central vision of patients with glaucoma. PLoS One. 2018;13:e0193465.

3. Rolle T, Dallorto L, Cafasso R, et al. Reading Ability in Primary Open-angle Glaucoma: Evaluation with Radner Reading Charts. Optom Vis Sci. 2019;96:55-61. Ophthalmol. 2018;29:135-40.

5. Glen FC, Crabb DP. Living with glaucoma: a qualitative study of functional implications 355 and patients' coping behaviours. BMC Ophthalmol. 2015;15:128. of gender and facial expression. Invest Ophthalm Vis Sci. 2018;59: 4921-28.

7. Itier RJ, Batty M. Neural bases of eye and gaze processing: The core of social cognition. Neurosci Biobehav Rev. 2009; 33:843-63. involved in gaze and emotional processing. Neurobiol Aging. 2016;48:182-94. during Dyadic Interactions. PLoS One.2015;10: e0136905. 
10. Torres-Marín J, Carretero-Dios H, Acosta A, \& Lupiáñez J. Eye Contact and Fear of Being Laughed at in a Gaze Discrimination Task. Front Psychol. 2017;8:1954.

11. Kaisler RE, Leder H. Combined Effects of Gaze and Orientation of Faces on Person Judgments in Social Situations. Front Psychol. 2017;8: 259.

12. Senju A, Vernetti A, Kikuchi Y, et al. Cultural background modulates how we look at other persons' gaze. Int J Behav Dev. 2012; 37:131-36.

13. Adams RB Jr, Kleck RE. Perceived gaze direction and the processing of facial displays of emotion. Psychol Sci. 2003;14:644-7.

14. Adams RB Jr, Kleck RE. Effects of direct and averted gaze on the perception of facially communicated emotion. Emotion. 2005;5:3-11.

15. Mason MF, Hood BM, Macrae CN. Look into my eyes: gaze direction and person memory. Memory. 2004;12:637-43.

16. Von Grünau M, Anston C. The detection of gaze direction: a stare-in-the-crowd effect. Perception. 1995; 24:1297-313.

17. Senju A, Hasegawa T. Do the upright eyes have it? Psychon Bull Rev. 2006;13:223-8.

18. Florey J, Clifford CW, Dakin SC, \& Mareschal I. Peripheral processing of gaze. J Exp Psychol: Hum Percept Perform. 2015;41:1084-94.

19. Crabb, DP, Viswanathan AC. Integrated visual fields: a new approach to measuring the binocular field of view and visual disability. Arch for Clin and Exp Ophthalm. 2005;243: 210-16.

20. Asaoka R, Crabb DP, Yamashita T et al. Patients have two eyes!: binocular versus better eye visual field indices. Invest Ophthalm Vis Sci. 2011;52:7007-11. 
387

400

401

402

403

404

405

406

407

408

409

21. George N, Driver J, Dolan RJ. Seen Gaze-Direction Modulates Fusiform Activity and Its Coupling with Other Brain Areas during Face Processing. NeuroImage. 2001;13:1102-12.

22. Itier RJ, Alain C, Kovacevic N, McIntosh AR. Explicit versus implicit gaze processing assessed by ERPs. Brain Res. 2007;1177:79-89.

23. Vida MD, Maurer D. A comparison of spatial frequency tuning for judgments of eye gaze and facial identity. Vis Res. 2015;112: 45-54.

24. Baseler HA, Harris RJ, Young AW, Andrews TJ. Neural Responses to Expression and Gaze in the Posterior Superior Temporal Sulcus Interact with Facial Identity. Cereb Cortex. $2014 ; 24: 737-44$.

25. Carlin JD, Calder AJ. The neural basis of eye gaze processing. Curr Opin Neurobiol. 2013;23:450-55.

26. Bernstein M, Yovel G. Two neural pathways of face processing: A critical evaluation of current models. Neurosci Biobehav Rev. 2015;55:536-46.

27. Ziaei M, Ebner NC, Burianov H. Functional brain networks involved in gaze and emotional processing. Europ J Neurosc. 2017;45:312-20.

28. Lawlor M, Danesh-Meyer H, Levin LA et al. Glaucoma and the brain: Trans-synaptic degeneration, structural change, and implications for neuroprotection. Surv Ophthalmol. 2018;63:296-306. 
29. Nuzzi R, Dallorto L, Rolle T. Changes of Visual Pathway and Brain Connectivity in Glaucoma: A Systematic Review. Front Neurosci. 2018; 12:363.

30. Yu L, Xie B, Yin X, et al. Reduced cortical thickness in primary open-angle glaucoma and its relationship to the retinal nerve fiber layer thickness. PLoS One. 2013;8:e73208.

31. Yu L, Yin, X, Dai, C, et al. Morphologic changes in the anterior and posterior sub-regions of $\mathrm{V} 1$ and $\mathrm{V} 2$ and the V5/MT+ in patients with primary open-angle glaucoma. Brain Res. $2014 ; 1588: 135-43$.

32. Yu L, Xie L, Dai C, et al. Progressive thinning of visual cortex in primary open-angle glaucoma of varying severity. PLoS One. 2015;10:e0121960.

\section{Grill-Spector K, Weiner KS, Kay K, Gomez J. The Functional Neuroanatomy of Human} Face Perception. Annu. Rev. Vis. Sci. 2017; 3:167-96.

\section{Slessor G, Phillips, LH, Bull R. Age-related changes in the integration of gaze direction} and facial expressions of emotion. Emotion. 2010;10:555-62.

35. Campbell A, Murray JE, Atkinson L, Ruffman T. Face Age and Eye Gaze Influence Older Adults' Emotion Recognition. J Gerontol B Psychol Sci Soc Sci. 2017;72:633-36.

36. Slessor G, Phillips LH, Bull R. Age-related declines in basic social perception: evidence from tasks assessing eye-gaze processing. Psychol Aging. 2008;23:812-22. 
431 37. Grillo LM, Wang DL, Ramachandran R, et al. The 24-2 Visual Field Test Misses Central

432 Macular Damage Confirmed by the 10-2 Visual Field Test and Optical Coherence

433 Tomography. Transl Vis Sci Technol. 2016; 14:15.

434 


\begin{tabular}{|c|c|c|c|c|c|c|c|c|c|}
\hline \multirow[t]{2}{*}{ PATIENTS } & \multirow[t]{2}{*}{ Age/Gender } & \multicolumn{2}{|c|}{ VA (LogMar) } & \begin{tabular}{|l|} 
VF \\
LE \\
\end{tabular} & MD 30-2 & \multirow[t]{2}{*}{ MD IVF } & \multicolumn{2}{|c|}{ VF MD 10-2 } & \multirow[t]{2}{*}{ MMSE/30 } \\
\hline & & LE & RE & LE & $\mathbf{R E}$ & & LE & RE & \\
\hline & $69 / M$ & 0.0 & 0.5 & -13 & -24 & -12 & -7 & -29 & 30 \\
\hline & $73 / F$ & 0.2 & 0.0 & -10 & -4 & -4 & $N$ & $\mathrm{~N}$ & 30 \\
\hline & $75 / \mathrm{F}$ & 0.1 & 0.1 & -9 & -5 & -4 & $N$ & $N$ & 30 \\
\hline & $53 / \mathrm{M}$ & 0.0 & 0.0 & $\mathrm{~N}$ & -28 & & $\mathrm{~N}$ & -20 & \\
\hline & $60 / M$ & 0.0 & 0.0 & -16 & -10 & -8 & $\mathrm{~N}$ & $\mathrm{~N}$ & 30 \\
\hline & $74 / F$ & 0.1 & 0.1 & -25 & -12 & -12 & -27 & -12 & 30 \\
\hline & $49 / \mathrm{M}$ & 0.4 & 0.0 & -12 & -3 & -3 & $\mathrm{~N}$ & $\mathrm{~N}$ & \\
\hline & $70 / F$ & 0.0 & 0.0 & -4 & -5 & -3 & $\mathrm{~N}$ & $\mathrm{~N}$ & 29 \\
\hline & $65 / F$ & 0.0 & 0.0 & -5 & -20 & -4 & $\mathrm{~N}$ & -24 & 28 \\
\hline 10 & $35 / F$ & 0.0 & 0.0 & -21 & -20 & -18 & $\mathrm{~N}$ & $\mathrm{~N}$ & \\
\hline 11 & $64 / F$ & 0.0 & 0.0 & -8 & -4 & -3 & $\mathrm{~N}$ & $\mathrm{~N}$ & 30 \\
\hline 12 & $80 / F$ & 0.0 & 0.0 & -3 & -7 & -3 & $\mathrm{~N}$ & $\mathrm{~N}$ & 30 \\
\hline 13 & $53 / M$ & 0.0 & 0.3 & -18 & -25 & -16 & -18 & -31 & \\
\hline 14 & $72 / M$ & 0.0 & 0.0 & -14 & -5 & -5 & $\mathrm{~N}$ & $\mathrm{~N}$ & 30 \\
\hline 15 & $85 / M$ & 0.1 & 0.3 & -4 & -24 & -4 & $\mathrm{~N}$ & -28 & 30 \\
\hline 16 & $62 / F$ & 0.0 & 0.0 & -9 & -6 & -2 & -19 & N & 30 \\
\hline 17 & $75 / M$ & 0.3 & 0.1 & -12 & -5 & -7 & $\mathrm{~N}$ & $\mathrm{~N}$ & 28 \\
\hline 18 & $52 / F$ & 0.0 & 0.1 & -4 & -4 & -3 & $\mathrm{~N}$ & N & \\
\hline 19 & $75 / M$ & 0.0 & 0.0 & -14 & -8 & -6 & $\mathrm{~N}$ & $\mathrm{~N}$ & 29 \\
\hline 20 & $75 / F$ & 0.0 & 0.3 & -12 & -5 & -3 & $\mathrm{~N}$ & $\mathrm{~N}$ & 30 \\
\hline 21 & $68 / M$ & 0.0 & 0.0 & -8 & -13 & -7 & $\mathrm{~N}$ & $\mathrm{~N}$ & 28 \\
\hline 22 & $70 / F$ & 0.1 & 0.0 & -9 & -8 & -3 & $\mathrm{~N}$ & $\mathrm{~N}$ & 30 \\
\hline 23 & $65 / F$ & 0.1 & 0.0 & -26 & -14 & -11 & -27 & -3 & 30 \\
\hline 24 & $40 / \mathrm{M}$ & 0.0 & 0.0 & -29 & -13 & & & $\mathrm{~N}$ & \\
\hline
\end{tabular}


Table 2

\begin{tabular}{|c|l|r|l|r|r|l|l|}
\hline AGE-MATCHED & Age/gender & MMSE/30 & Binoc VA & & YOUNG & Age/gender & Binoc VA \\
\hline 1 & $55 / \mathrm{F}$ & & 0.0 & & 1 & $29 / \mathrm{M}$ & 0.0 \\
\hline 2 & $56 / \mathrm{F}$ & & 0.0 & & 2 & $28 / \mathrm{M}$ & 0.0 \\
\hline $346 / \mathrm{M}$ & & 0.0 & & 3 & $29 / \mathrm{F}$ & 0.0 \\
\hline $467 / \mathrm{F}$ & 30 & 0.0 & & 4 & $23 / \mathrm{M}$ & 0.0 \\
\hline 5 & $50 / \mathrm{F}$ & & 0.0 & & 5 & $21 / \mathrm{F}$ & 0.0 \\
\hline 6 & $51 / \mathrm{F}$ & & 0.0 & & 6 & $28 / \mathrm{M}$ & 0.0 \\
\hline 7 & $52 / \mathrm{M}$ & & 0.0 & & 7 & $26 / \mathrm{M}$ & 0.0 \\
\hline $876 / \mathrm{F}$ & 30 & 0.0 & & 8 & $28 / \mathrm{M}$ & 0.0 \\
\hline $935 / \mathrm{F}$ & & 0.0 & & 9 & $26 / \mathrm{M}$ & 0.0 \\
\hline 10 & $75 / \mathrm{M}$ & 28 & 0.0 & & 10 & $26 / \mathrm{M}$ & 0.0 \\
\hline $1173 / \mathrm{M}$ & 30 & 0.0 & & 11 & $30 / \mathrm{M}$ & 0.0 \\
\hline 12 & $71 / \mathrm{F}$ & 30 & 0.0 & & 12 & $27 / \mathrm{M}$ & 0.0 \\
\hline 13 & $67 / \mathrm{M}$ & 30 & 0.0 & & 13 & $28 / \mathrm{F}$ & 0.0 \\
\hline 14 & $74 / \mathrm{F}$ & 30 & 0.0 & & 14 & $29 / \mathrm{M}$ & 0.0 \\
\hline 15 & $64 / \mathrm{F}$ & 30 & 0.0 & & 15 & $25 / \mathrm{F}$ & 0.0 \\
\hline 16 & $64 / \mathrm{M}$ & 30 & 0.0 & & 16 & $25 / \mathrm{M}$ & 0.0 \\
\hline $1756 / \mathrm{F}$ & & 0.0 & & 17 & $29 / \mathrm{M}$ & 0.0 \\
\hline 18 & $52 / \mathrm{M}$ & & 0.0 & & 18 & $28 / \mathrm{F}$ & 0.0 \\
\hline 19 & $67 / \mathrm{M}$ & 28 & 0.0 & & 19 & $29 / \mathrm{M}$ & 0.0 \\
\hline $2064 / \mathrm{M}$ & 29 & 0.0 & & 20 & $28 / \mathrm{F}$ & 0.0 \\
\hline $2163 / \mathrm{F}$ & 30 & 0.0 & & & & \\
\hline
\end{tabular}


Figure 1
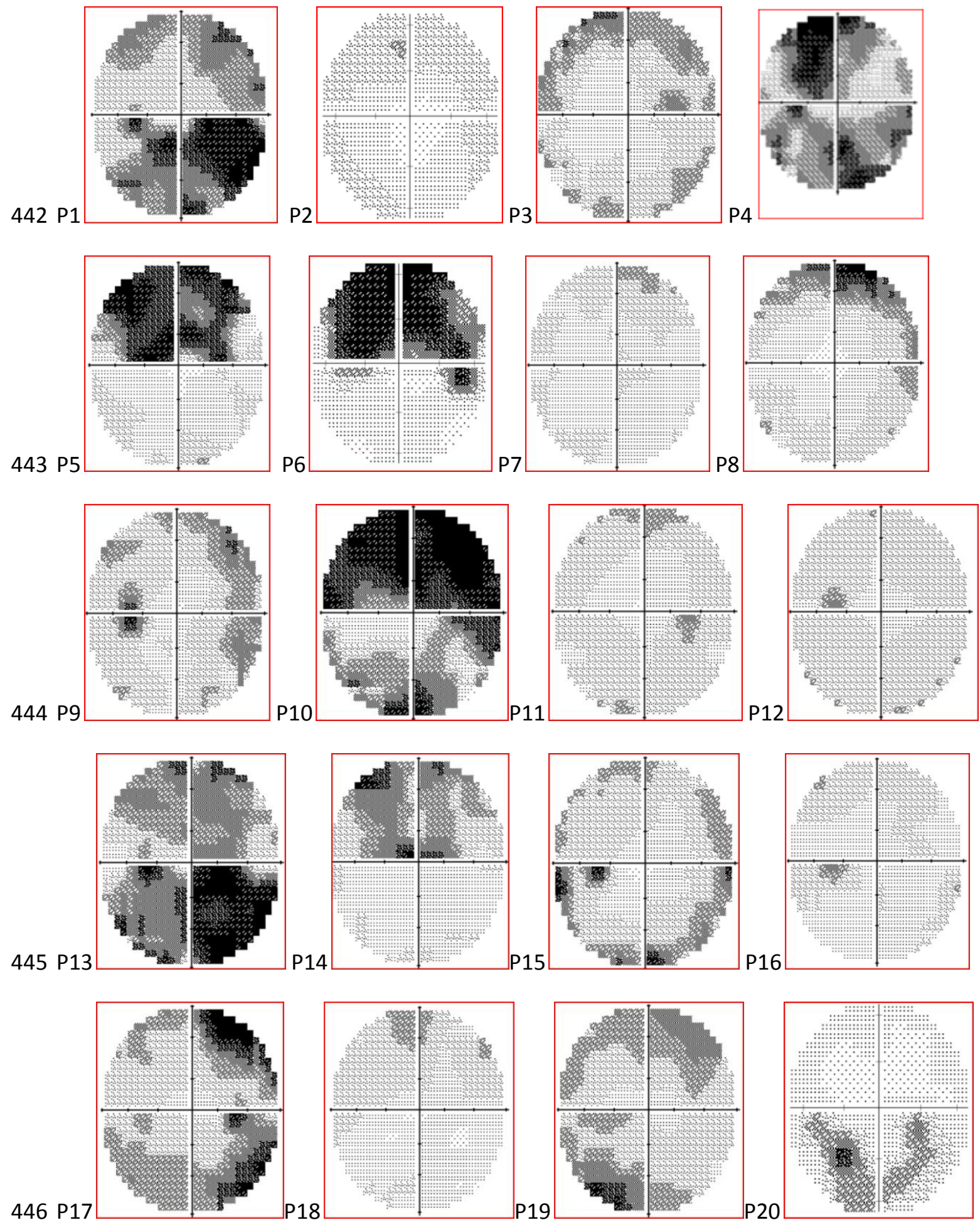


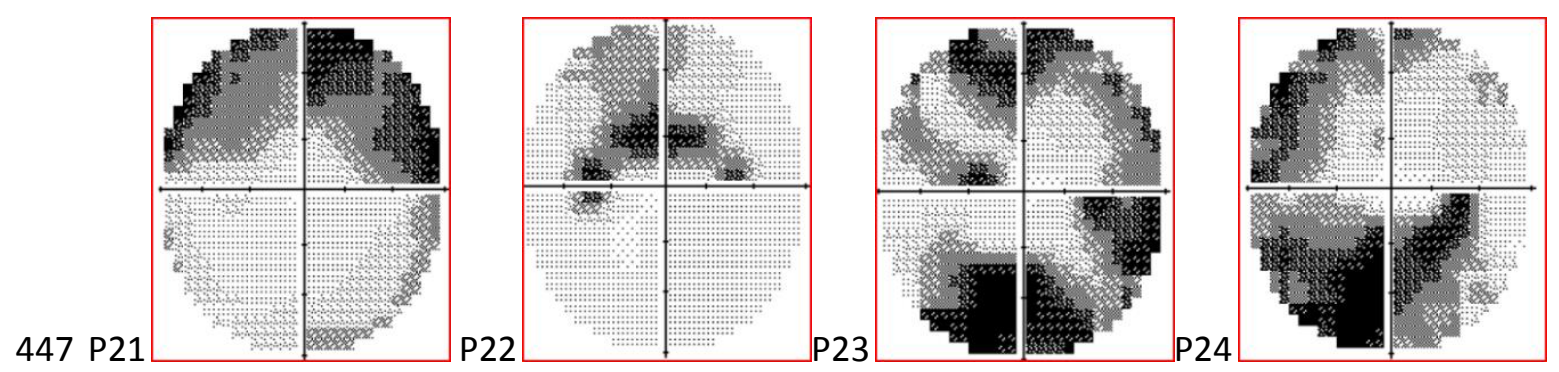

448 


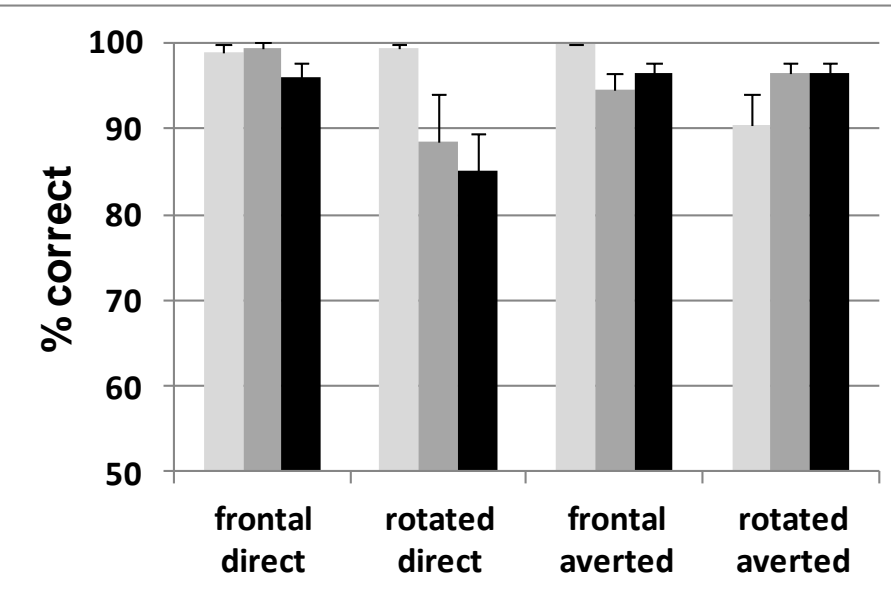

Young

age matched

- patients

Face orientation and Gaze direction

A

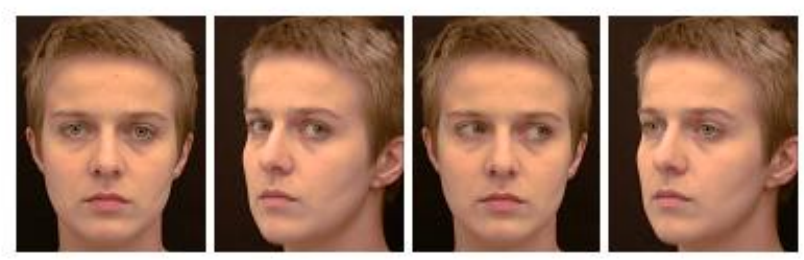

451

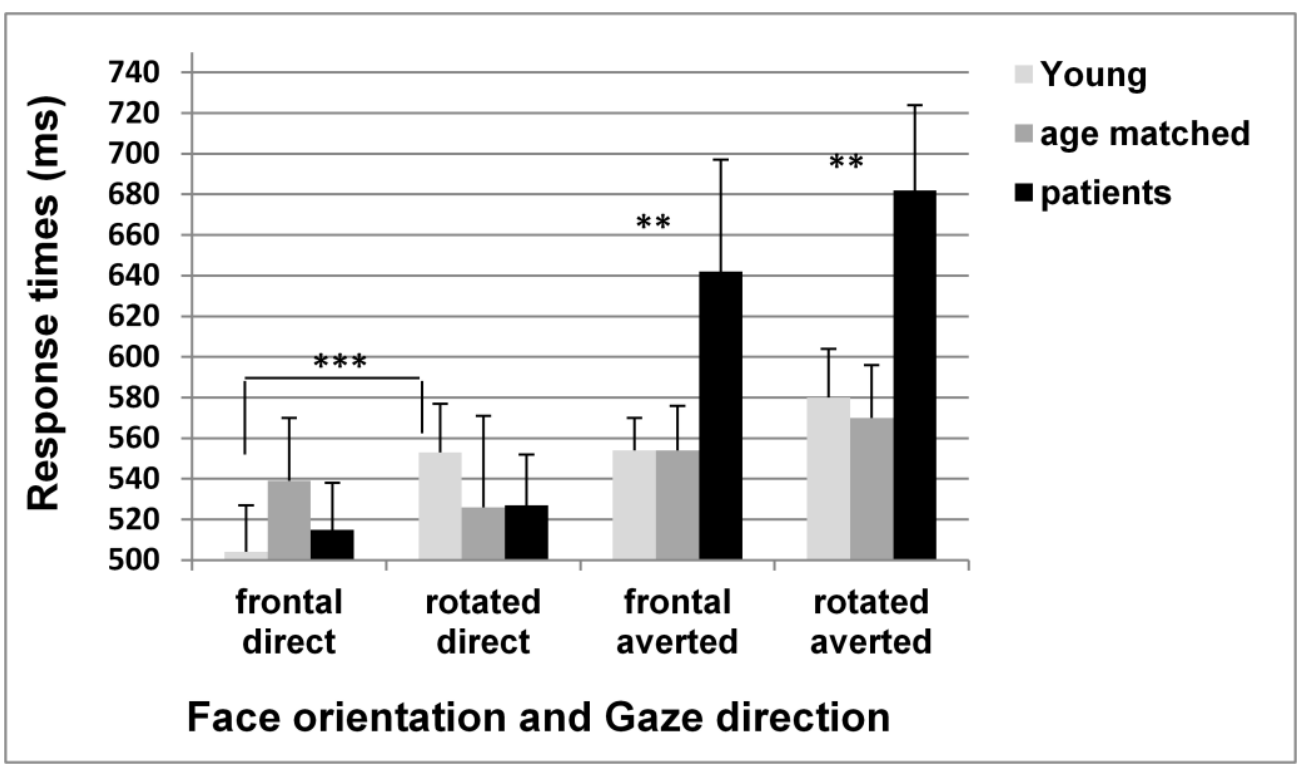

453 